\title{
USO DE CAROÇOS DE AÇAIZEIRO, PALMITEIRO E FIBRAS DE PERICARPO DE COCO VERDE COMO SUBSTRATOS PARA A ORQUÍDEA OLHO DE BONECA
}

\author{
Giorgini Augusti Venturieri'; Thiago Nunes Teixeira²; Marina Juliana Batista Barwinski ${ }^{3}$ \\ 'Universidade Federal de Santa Catarina (UFSC), Florianópolis, Santa Catarina, Brasil, \\ giorgini.venturieri@ufsc.br \\ 2UFSC, Florianópolis, Santa Catarina, Brasil, thiagopakua@hotmail.com \\ 3UFSC, Florianópolis, Santa Catarina, Brasil, marinajbatista@gmail.com
}

RESUMO: A orquídea olho de boneca (Dendrobium nobile), é uma espécie ornamental originária do Sudeste Asiático, de fácil cultivo, com floração abundante durante a primavera, sendo hoje a orquídea de menor preço do mercado e por isto uma das mais comercializadas. Os derivados do caule do xaxim (Dicksonia sellowiana) têm sido reputados como os melhores substratos para orquídeas, mas devido a sua sobrexploração, a sua coleta e uso foram proibidos e, desde então, substratos alternativos vêm sendo buscados. No presente trabalho foi estudada a influência de diferentes substratos na sobrevivência, altura, número de brotos laterais ("Keikes") e no vigor da orquídea olho de boneca. Os tratamentos foram: 1) pó de xaxim - considerado como a testemunha; 2) pó de xaxim mais caroços de palmiteiro na proporção de 1:1; 3) fibras de pericarpo de coco verde (Coccus nucifera) lavadas; 4) fibras de pericarpo de coco verde; 5) caroços secos de palmiteiro (Euterpe edulis); 6) caroços secos de açaizeiro (E. oleracea). Os substratos feitos com caroços de palmiteiro e fibras de pericarpo de coco verde lavadas não diferiram estatisticamente dos substratos formulado somente com o pó de xaxim ou a mistura de pó de xaxim: caroços de palmiteiro e, portanto, podem ser usados como alternativos ao pó de xaxim. Devido à elevada disponibilidade de cocos verdes, caroços de açaizeiro e de palmiteiro, sugere-se que melhorias nas formulações e no manejo das plantas cultivadas sobre os substratos que os usem como base, continuem sendo buscadas. São sugeridas: a mescla com outros materiais e sistemas de adubação e de irrigação, para melhorarem o seu desempenho e o uso de espécies de orquídeas mais adaptadas a tais substratos.

PALAVRAS CHAVE: Formulação, Substrato alternativo, Xaxim. 


\title{
USE OF ASSAI SEEDS, PALM HEART AND GREEN COCONUT PERICARP FIBERS AS SUBSTRATES FOR THE NOBLE DENDROBIUM ORCHID
}

\begin{abstract}
The noble dendrobium orchid (Dendrobium nobile), is an ornamental species native of Southeast Asia, easy to grow, with profuse blossoming during springs, been nowadays the cheapest orchid in the market and so, one of the most commercialized. Tree fern (Dicksonia sellowiana) fibers from their trucks have been reputed as the best substrate for orchids, but due to its overexploitation, its gathering from nature and use were prohibited and so, alternative substrates have been fetched. In the present work was studied the influence of different substrates on survival, lateral shoot emission ("Keykes"), and vigor of the orchid noble dendrobium (Dendrobium nobile). Treatments applied were: 1) tree fern dust - considered as a control treatment; 2) mix of tree fern dust with dried seed of palm heart at the proportion of $1: 1 ; 3)$ washed pericarp fibers from green coconut (Coccus nucifera); 4) pericarp fibers from green coconut; 5) dried seed of palm heart (Euterpe edulis); and 6 ) dried seed of assai (E, oleracea).The substrates made with washed pericarp fibers from green coconut and pericarp fibers from green coconut did not differ statistically from the substrates formulated with the tree fern dust only or a mixture of tree fern dust: dried seed of palm heart, and so, can be used as alternatives to tree fern dust. Due to high availability of green coconuts, assai and palm heart seeds, it is suggested that improvements, in the formulation and in the management of plants grown on substrates that use them as a base, continue to be fetched. They are suggested: mixtures with other materials, conjugated to irrigation and fertilization systems to improve their performance and the search for more adapted orchid species to such substrates.
\end{abstract}

KEYWORDS: Alternative substrate, Formula, Xaxim.

\section{EL USO DE SEMILLAS DE PALMA DE ASAÍ, PALMITO Y FIBRAS DI PERICARPIO DE COCO VERDE COMO SUSTRATO DE ORQUÍDEA DENDROBIUM}

RESUMEN: La orquídea dendrobium (Dendrobium nobile), es una especie ornamental nativa del Sureste de Asia, fácil de cultivar, con floración abundante durante la primavera siendo hoy la orquídea más barata en el mercado y por lo tanto, una de las más comercializadas. Derivados del tallo del helecho arborescente (Dicksonia sellowiana) son conocidos como el mejor sustrato para orquídeas, pero debido a su sobreexplotación, su recolección y uso fueron prohibidos, y desde entonces se ha buscado sustratos

Agroecossistemas, v. 7, n. 1, p. 18 - 26, 2015, ISSN online 2318-0188 
alternativos. En este trabajo se estudió la influencia de diferentes sustratos en la mortalidad, brotes laterales ("Keikes") y vigor de la orquídea dendrobium. Los tratamientos fueron: 1) polvo de helecho considerado como el testigo; 2) mezcla de polvo de helecho más semillas de palmito en la proporción de 1:1; 3) fibras di pericarpio de coco verde lavadas; 4) fibras di pericarpio de coco verde (Coccus nucifera); 5) semilla seca de palmito seco (Euterpe edulis); y 6) semilla seca de palmera de asaí (E. oleracea). Los sustratos hechos con semilla seca de palmito y fibras di pericarpio de coco verde lavadas no difirieron estadísticamente de los sustratos formulados solamente con el polvo de helecho o la mezcla de polvo de helecho: semilla seca de palmito y, por lo tanto, pueden ser usados como alternativa al polvo de helecho. Debido a la elevada disponibilidad de cocos verdes, semillas de la palmera de asaí, se sugiere que ajustes en las formulaciones y el manejo de las plantas cultivadas sobre los sustratos que los usen como base, sigan siendo buscadas. Se sugieren: el uso de mezclas con otros materiales y sistemas asociados de fertilización y riego, para permitir su uso y calidad y la búsqueda de las especies de orquídeas más adaptadas a tales sustratos.

PALABRAS CLAVE: Formulación, Helechos, substrato alternativo.

\section{INTRODUÇÃO}

A orquídea olho de boneca (Dendrobium nobile Lindl.) é originária do Sudeste Asiático (BAKER; BAKER, 1996). As suas flores, de diâmetro entre três a oito centímetros, são de cores muito variadas, porém, com a característica marcante da presença de uma mancha escura no fundo do labelo, o que a faz parecer com um olho (Figura 1). Cultivares melhorados e híbridos envolvendo esta espécie começaram a ser cultivadas comercialmente no Brasil no início dos anos 90 , sendo hoje a orquídea de menor preço do mercado e por isto uma das mais comercializadas. É uma das raras orquídeas de fácil cultivo e que pode ser propagada comercialmente por estaquia (a maioria o é por cultura de tecidos), em um processo simples, desenvolvido para ser feito pelos próprios agricultores, tornando-os autosuficientes para este insumo (VENTURIERI; PICKSCIUS, 2013; SOCCOL et al., 2017). 
Figura 1. Aspecto geral da flor da olho-de-boneca (A) e dos vasos que compuseram o experimento (B).
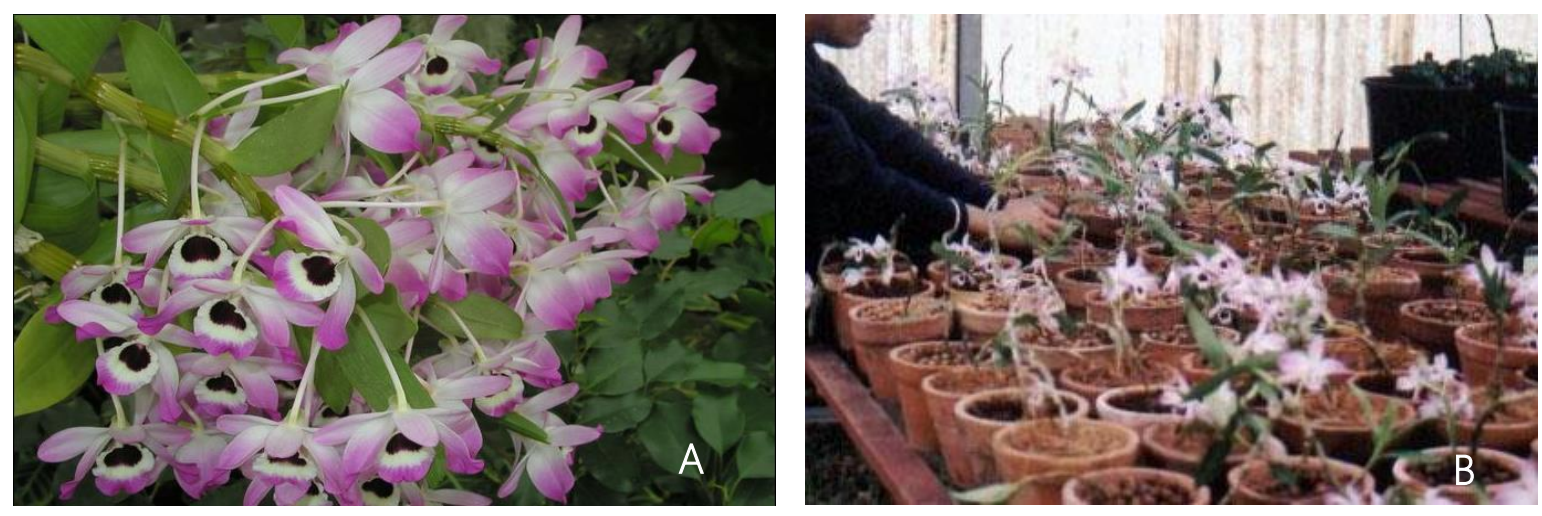

Esta espécie vegeta bem de planícies até altitudes de 2000 m, suporta temperaturas de até $-1{ }^{\circ} \mathrm{C}$, mas para um bom desenvolvimento vegetativo precisa de temperaturas acima de $22^{\circ} \mathrm{C}$; pode ser cultivada na maioria dos substratos indicados para orquídeas e as baixas temperaturas induzem a produção de flores que desabrocham do fim do inverno até o início do verão (BAKER; BAKER, 1996).

O xaxim é o substrato preferido entre os cultivadores de orquídeas brasileiros. É extraído das raízes adventícias de algumas samambaias da família Dicksoniaceae e Cyatheaceae
(DEMATTÊ; DEMATTÊ, 1996) sendo a espécie mais usada a samambaia-açu ou xaxim (Dicksonia sellowiana (Presl.) Hooker) (FARIA et al., 2001). Os xaxins são vendidos como "vasos", "fibra", e como "pó", sendo este último um subproduto derivado da fabricação dos vasos e da fibra. A exploração desenfreada dos estoques naturais do xaxim no Brasil levou a proibição do seu corte em todo o território nacional (resolução Conselho Nacional do Meio Ambiente n. 278/2001) e, até o presente, não se tem conhecimento do cultivo comercial desta planta que possa tirá-la 
da lista de espécies ameaçadas de extinção.

Substratos para orquídeas são basicamente feitos com matéria orgânica podendo ser misturado com algum outro componente para mitigar deficiência na aeração (como pedras e carvão vegetal) e/ou na retenção de água (como o pó de coco, esfagno, vermiculita, perlita e até esponja sintética). As pedras, quando adicionadas aos substratos, servem ainda para que as orquídeas fixem as suas raízes e para dar mais peso aos vasos para que não tombem com facilidade. A aeração e a hidratação de um substrato podem ainda ser influenciadas pelo tipo de vaso usado (de plástico, barro ou engradados); adubações; regime de irrigação; e proteção (ou não) da chuva. Combinações de todos estes fatores podem ser feitas para adequar o substrato às necessidades da espécie que se deseja cultivar. Devido a esta flexibilidade, a escolha de um substrato, na maioria das vezes, é uma decisão equilibrada entre: preço, disponibilidade no mercado dos seus componentes e a qualidade das plantas conseguidas com o seu uso. Quando um componente para a formulação de substratos é muito barato e abundante ele é preferido para servir de base que, com a adição de outros componentes que o melhore, torne o seu uso aceitável.

No Brasil, embora os componentes capazes de serem usados para fazer boas formulações de substrato sejam geralmente fáceis de serem encontrados, substratos específicos para orquídeas praticamente não existem no mercado.

O vertiginoso aumento do consumo do suco do açaí da Amazônia (Euterpe oleracea Mart.) fez com que fossem disponibilizadas quantidades expressivas de caroços, geralmente inaproveitados. O mesmo tem acontecido, mais recentemente, com o palmiteiro (E. edulis Mart.), uma espécie das regiões Sudeste e Sul do Brasil, cujos frutos, por 
terem sabor e aspecto idênticos ao do açaí da Amazônia, têm sido usados para preparar o cognominado "açaí da mata atlântica" e comercializado como aquele. A produção brasileira de frutos do açaizeiro, estimada para 2013, foi de 1.118.870 T, sendo o estado do Pará, o maior produtor nacional, responsável por 74\% deste total (TAVARES; HOMMA, 2015). Considerando que, $87,5 \%$ deste volume corresponde a caroços e fibras gerados após o processamento do fruto (RODRIGUES et al., 2006), somente para este estado, ter-se-iam 722.323,9 T de resíduos que poderiam vir a ser usados como substratos para plantas. Outra fonte abundante de matéria prima para substratos é o coco (Cocos nucifera L.), que ainda verde, após o consumo da sua água, vem sendo espalhado pelas praias brasileiras. Tanto as sementes do açaí como as do coco são geralmente jogadas fora formando montes em várias cidades, transformando em um estorvo para as prefeituras. As sementes do palmiteiro ainda não chegaram a este nível, mas, já que o açaí da mata atlântica vem se popularizando (TREVISAN et al., 2015), resíduos expressivos também poderão vir a ser formados.

No presente trabalho foram estudados substratos alternativos ao pó de xaxim, baseados em resíduos, abundantes e ainda considerados como lixo nas cidades (no caso os cocos verdes, caroços de palmiteiro e de açaizeiro) no crescimento das orquídeas olho de boneca ( $D$. nobile Lindl.).

\section{MATERIAL E MÉTODOS}

O experimento foi implantado em novembro de 2002, em Florianópolis, Santa Catarina (27050's, 483' W), em casa de vegetação, coberta com plástico leitoso com capacidade de bloqueio de $60 \%$ da luz incidente. Plantas de D. nobile Lindl., derivadas do brotamento lateral de caules ("keikes"), com tamanho aproximado de $10 \mathrm{~cm}$, foram plantadas em vasos de barro, com $11 \mathrm{~cm}$ de diâmetro (uma planta por vaso), contendo diferentes substratos 
(tratamentos): 1) pó de xaxim (D. sellowiana (Presl.) Hooker); 2) mistura de pó de xaxim mais caroços de palmiteiro na proporção de 1:1 (v/v); 3) fibras de pericarpo de coco verde lavado (deixado de molho em água, por 4 dias, com a renovação diária da água); 4) fibras de pericarpo de coco verde (Coccus nucifera L.); 5) caroços secos de palmiteiro ( $E$. edulis Mart.); e 6) caroços secos de açaizeiro (E. oleracea Mart.). Os caroços destes dois últimos substratos citados foram previamente secos, por 1 mês, para que não germinassem mais. $\bigcirc$ delineamento experimental foi inteiramente casualizado.

Os parâmetros medidos foram: a) sobrevivência e altura das plantas após 1, e 2 anos; número de brotações laterais ("keikes") e vigor das plantas aos dois anos após o início do experimento. Para esta última foram atribuídas notas de 1 a 3 sendo: 1 para plantas fracas, com o

${ }^{1}$ Ouro Verde é um produto da Empresa Paulista de Produtos Químicos - Ltda contendo: N (6\%); $\mathrm{P}_{2} \mathrm{O}_{5}(6 \%) ; \mathrm{K}_{2} \mathrm{O}(8 \%) ; \mathrm{Mg}(0,5 \%) ; \mathrm{S}(0,5 \%) ; \mathrm{B}$ (0,03\%); Zn (0,05\%); Fe (0,1\%); Mn (03\%). caule aproximadamente cilíndrico e levemente amarelecido; 2 para plantas sadias, com o caule com o diâmetro maior na base da planta; e 3, para plantas com crescimento vigoroso e caule com o diâmetro maior no meio da planta.

○ parâmetro sobrevivência foi calculado em 4 parcelas por tratamento, sendo cada uma com 6 plantas. Os demais parâmetros foram tomados de forma individual, portanto considerando cada planta uma unidade amostral, tomados de 13 a 21 plantas por tratamento.

As plantas receberam adubação foliar quinzenal básica (2 $\mathrm{ml} \mathrm{L}^{-1}$ de adubo Ouro Verde $^{\circledR 1}$ 6-6-8, mais $2 \mathrm{ml} \mathrm{L}^{-1}$ de SupaPotássio ${ }^{\circledR 2}$, associada a um fungicida (2 $\mathrm{ml} \mathrm{L}{ }^{-1}$ de Manzate ${ }^{\circledR 3}$ ).

Os parâmetros avaliados foram submetidos à análise de variância usando o modelo Linear Generalizado

\footnotetext{
25upa - Potássio é um produto da Agrinchem do Brasil Ltda contendo: $\mathrm{K}_{2} \mathrm{O}$ (23\%) e $\mathrm{SiO}$ (20\%). ${ }^{3}$ Manzate é um produto da DuPont, contendo como princípio ativo o Mancozeb.
} 
(ANOVA-GLM), e as médias comparadas entre si pelo teste de Fischer para $\alpha=$ 0,05 (MYERS et al. 2010).

As médias de cada parâmetro alcançadas em cada tratamento foram transformadas em unidades de escore padrão (escore "Z"), para que se tornassem adimensionais, e apresentadas em um gráfico. Isto foi feito para ter-se uma visão comparativa dos tratamentos com base na ordem de classificação de todos os parâmetros avaliados. Os tratamentos foram submetidos a análise de Kruskal-Wallis, usando-se como escores as posições relativas dos parâmetros por tratamento. Os tratamentos forma comparados, par a par, pelo teste de Dunn para $\alpha=0,05$.

A comparação entre plantas que sobreviveram no primeiro ano com as do segundo ano foi feita através do teste Wilcoxon para amostras pareadas (DYTHAM, 2010).
Os dados foram analisados com 0 auxílio do programa Statistica (Statsoft, Inc., Tulsa, USA).

\section{RESULTADOS E DISCUSSÃO}

A sobrevivência no primeiro ano foi significativamente diferente entre os tratamentos $(p=0,025)$, sendo o pó de xaxim o que teve a maior média de sobrevivência. $\bigcirc$ pó de xaxim, embora não tenha diferido estatisticamente das fibras de pericarpo de coco verde e das fibras de pericarpo de coco verde lavadas, foi significativamente superior aos tratamentos mistura de pó de xaxim: caroços de palmiteiro, caroços de açaizeiro, e caroços de palmiteiro. Estes três últimos não diferiram estatisticamente entre si. As fibras de pericarpo de coco verde lavadas, das fibras de pericarpo de coco verde, a mistura de pó de xaxim: caroços de palmiteiro, e caroços de açaizeiro, não diferiram significativamente entre si (Tabela 1). 
Tabela 1. Médias observadas para os parâmetros avaliados. Valores seguidos da mesma letra não diferiram estatisticamente entre si para $\alpha=0,05$.

\begin{tabular}{|c|c|c|c|c|c|c|c|c|c|}
\hline Tratamentos & $\begin{array}{l}\text { Sobrevivência } \\
\text { - ano } 1 \text { (\%) }\end{array}$ & $\begin{array}{l}\text { Sobrevivência } \\
\text { - ano } 2(\%)\end{array}$ & $\begin{array}{l}\text { Altura da planta - } \\
\text { ano } 1(\mathrm{~cm})\end{array}$ & $\mathrm{N}^{*}$ & $\begin{array}{l}\text { Altura } \\
\text { da } \\
\text { planta } \\
- \text { ano } \\
2 \text { (cm) }\end{array}$ & N & $\begin{array}{l}\text { Número } \\
\text { de } \\
\text { brotos } \\
\text { laterais } \\
\text { (N) }\end{array}$ & N & $\begin{array}{l}\text { Vigor } \\
\text { (nota) }\end{array}$ \\
\hline Pó de xaxim & $87,5 \mathrm{a}$ & $87,5 \mathrm{a}$ & $21,2 \mathrm{a}$ & 21 & $23,5 \mathrm{a}$ & 21 & $0,04 \mathrm{a}$ & 21 & $7,14 \mathrm{a}$ \\
\hline $\begin{array}{l}\text { Mistura de } \\
\text { pó de xaxim } \\
\text { : caroços de } \\
\text { palmiteiro }\end{array}$ & $58,33 b c$ & 54,2 bc & $20,3 a b$ & 13 & $14,7 \mathrm{~b}$ & 13 & $0,31 \mathrm{a}$ & 13 & $5,8 \mathrm{~b}$ \\
\hline $\begin{array}{l}\text { Caroços de } \\
\text { palmiteiro }\end{array}$ & $45,83 \mathrm{c}$ & $41,7 \mathrm{C}$ & $21,1 \mathrm{ab}$ & 10 & $13,9 \mathrm{~b}$ & 10 & $0,30 \mathrm{a}$ & 10 & $4,9 \mathrm{~b}$ \\
\hline $\begin{array}{l}\text { Fibras de } \\
\text { pericarpo } \\
\text { de coco } \\
\text { verde } \\
\text { lavadas }\end{array}$ & $75,0 \mathrm{ab}$ & $58,3 \mathrm{bc}$ & $18,6 a b$ & 14 & $8,7 b$ & 14 & $0,21 \mathrm{a}$ & 14 & $5,9 b$ \\
\hline $\begin{array}{l}\text { Fibras de } \\
\text { pericarpo } \\
\text { de coco } \\
\text { verde }\end{array}$ & $75,0 a b$ & $70,8 \mathrm{ab}$ & $18,9 a b$ & 17 & $11,2 \mathrm{~b}$ & 17 & $0,11 \mathrm{a}$ & 17 & $5,6 b$ \\
\hline $\begin{array}{l}\text { Caroços de } \\
\text { açaizeiro }\end{array}$ & $55,56 \mathrm{bc}$ & 55,56 bc & $15,8 \mathrm{~b}$ & 10 & $10,4 \mathrm{~b}$ & 10 & $0,00 \mathrm{a}$ & 10 & $4,8 \mathrm{~b}$ \\
\hline
\end{tabular}

Obs. * número de plantas avaliadas.

A sobrevivência no segundo ano foi significativamente diferente entre os tratamentos $(p=0,025)$. Assim como no primeiro ano, o pó de xaxim manteve a maior média de sobrevivência. $\bigcirc$ pó de xaxim foi significativamente superior as: fibras de pericarpo de coco verde. As fibras de pericarpo de coco verde lavadas, caroços de açaizeiro, fibras de pericarpo de coco verde, e a mistura de pó de xaxim: caroços de palmiteiro, não diferiram significativamente entre si. As fibras de pericarpo de coco verde lavadas, os caroços de açaizeiro, a mistura de pó de xaxim: caroços de palmiteiro, e caroços de palmiteiro, não diferiram significativamente entre si. Tanto no primeiro como no segundo 
ano o pó de xaxim foi o substrato que apresentou maior sobrevivência (Tabela 1).

A sobrevivência avaliada no segundo ano (média = 61\%) foi significativamente inferior à observada no primeiro (média =66\%) $(p=0,028$ para $\mathrm{N}=24)$ devido à diminuição da sobrevivência em quatro dos seis substratos sendo o pó de xaxim e caroço de açaizeiro os que se mantiveram inalterados (Tabela 1).

A altura da planta no primeiro ano nos diferentes substratos diferiu estatisticamente $(p \cong 0,00)$ (Tabela 1$)$ sendo a mais elevada no pó de xaxim, que só diferiu estatisticamente dos caroços de açaizeiro, que teve a menor altura. Os demais substratos não diferiram estatisticamente entre si e foram iguais tanto ao pó de xaxim quanto aos caroços de açaizeiro (Tabela 1).

As alturas das plantas no segundo ano, nos diferentes substratos, diferiram estatisticamente $(p \cong 0,05)$ sendo a mais elevada conseguida sobre o substrato pó de xaxim. Para esta variável não houve diferença significativa entre os demais substratos, que em média, atingiram cerca da metade da altura conseguida com o pó de xaxim (Tabela 1).

O número de brotos laterais formados por tratamento não mostrou diferença significativa entre tratamentos $(p=0,05)$ (Tabela 1). As brotações laterais do tipo avaliado ("Keikes") não são benéficas para quando se deseja produzir uma planta para o consumidor final. No presente experimento variou de o a 0,31, ou seja poucos chegaram a ser formados, portanto, não configurando um problema para a produção de flores. No entanto, o nível de significância esteve próximo de ser atingido, o que pode ser considerado como um indicativo de que os substratos possam estar, em realidade, influenciando nesta variável.

O vigor diferiu significativamente entre substratos $(p=0,05)$ tendo sido mais elevado para o pó de xaxim que se diferenciou dos demais que, em 
conjunto, não mostraram diferenças

significativas entre si. Para a maioria dos

parâmetros avaliados o pó de xaxim teve as maiores médias, sendo para dois deles (sobrevivência no segundo ano, e vigor), superiores a todos os demais substratos.

A análise de um substrato, por parâmetro avaliado, é útil para que sejam evidenciadas deficiências e sugeridas estratégias para mitigá-las. No entanto, a comparação envolvendo todos os parâmetros dá uma visão global do desempenho de cada substrato em relação aos demais estudados como pode ser visto na Figura 2.

Figura 2. Comparação entre substratos com base em todos os parâmetros avaliados. Os substratos seguidos da mesma letra não diferem entre si para $\alpha=0,5$.

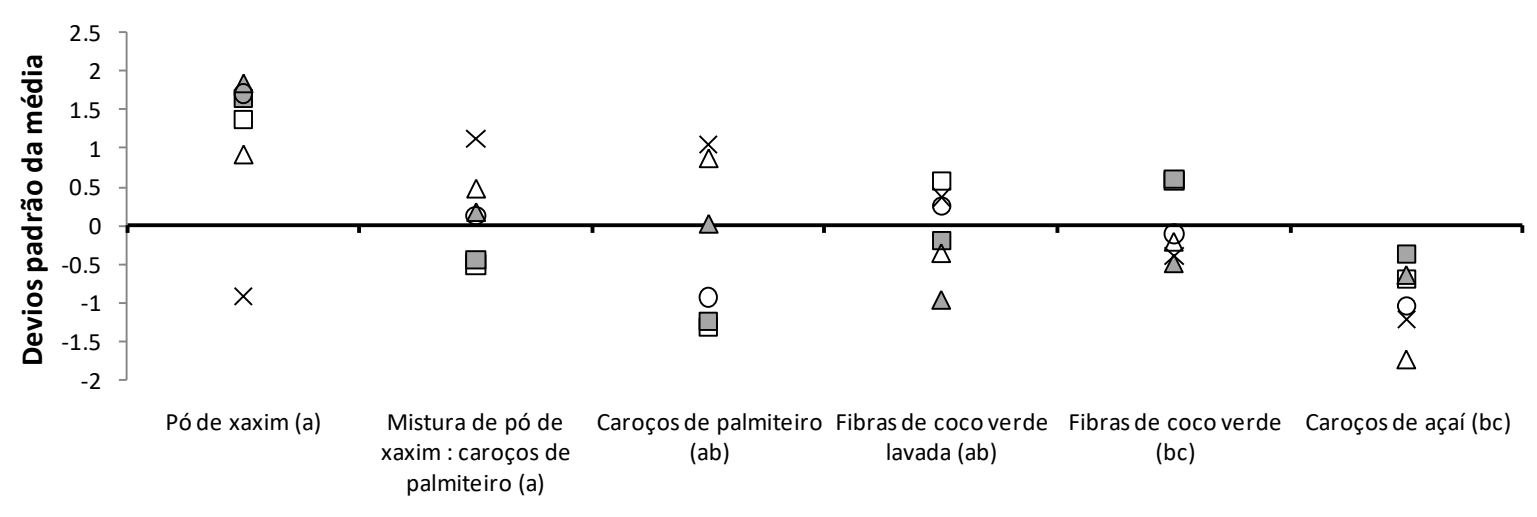

Substratos

$\square$ Sobrevivência (primeiro ano)

$\triangle$ Altura da planta (segundo ano) $\square$ Sobrevivência (segundo ano)

$\times$ Número de brotos laterais $\triangle$ Altura da planta (primeiro ano)

OVigor
O substrato feito com pó de xaxim apresentou maior desempenho, mas, ele não diferiu estatisticamente dos substratos feito com: a mistura de pó de xaxim: caroços de palmiteiro; caroços de palmiteiro; fibras de pericarpo de coco verde lavadas. Os piores desempenhos ficaram com os substratos elaborados 
com fibras de pericarpo de coco verde e caroços de açaizeiro, que não diferiram estatisticamente dos caroços de palmiteiro, e fibras de pericarpo de coco verde lavadas e das fibras de pericarpo de coco verde.

Amaral et al. (2010) avaliaram o uso de fibra de coco sob duas formas: 1) industrializada e 2) não industrializada no crescimento do híbrido Phalaenopsis RJ 343, sob quatro sistemas de adubação. No mesmo trabalho também foi avaliado o híbrido Phalaenopsis RJ 84-2 nos dois substratos, com as mesmas doses de adubo mineral, porém associado e não associado à aplicação foliar de Aminon ${ }^{\circledR}$. O uso do Aminon ${ }^{\circledast}$ não teve efeito sobre o crescimento dos híbridos. O aumento da adubação mineral promoveu aumento de crescimento, exceto de raízes. A fibra de coco não industrializada promoveu maior crescimento das plantas do híbrido RJ 343, enquanto a industrializada promoveu maior crescimento do híbrido RJ 84-2. Os autores concluíram que ambos os substratos podem ser utilizados no cultivo de híbridos de Phalaenopsis, desde que consideradas as exigências particulares de cada material vegetal.

Assis et al. (2005) compararam a eficiência de substratos alternativos ao xaxim desfibrado feitos com mesocarpo de coco industrializado (possivelmente provenientes de cocos maduros), associado ao uso de adubo foliar e à adubação orgânica, no desenvolvimento de mudas de $D$. nobile. A maioria dos tratamentos alternativos foi melhor que o xaxim desfibrado.

Assis et al. (2008), em experimento similar, porém cultivando Oncidium baueri Lindl., demonstraram que os substratos feitos com coco em pó e a mistura de coco desfibrado com coco em pó foram tão eficientes ao desenvolvimento das plantas quanto o xaxim desfibrado.

Yamakami et al. (2006) comparou o crescimento de mudas do hibrido Cattleya labiata $\times$ C. forbesiie Lindl. em 
fibra de coco, entre outros, com o xaxim

desfibrado. Os resultados não mostraram diferenças estatísticas para seis, dos sete parâmetros avaliados, portanto, a fibra de coco foi considerada como substrato alternativo ao xaxim desfibrado.

Colombo et al. (2005) estudaram a aclimatização na fase ex vitro de plântulas da orquídea híbrida Cattleya Chocolate Drop $\times$ (C. guttata $\times$ Laelia tenebrosa) nos substratos de: 1) pó de coco; 2) fibra de coco; 3) xaxim desfibrado; e 4) esfagno, sob dois sistemas de irrigação (manual e intermitente automatizado). Em termos gerais os autores indicam o pó de coco, em sistema de irrigação intermitente, como o melhor sistema para a climatização do híbrido estudado.

Sorace et al. (2009), avaliaram a eficiência de oito substratos tendo como base o xaxim desfibrado; casca de arroz carbonizada; fibra de coco; casca de pinus; e combinações entre eles, na aclimatação de mudas da orquídea C. intermedia x Hadrolaelia purpurata, provenientes de cultivo in vitro. Os autores concluíram que todos os substratos testados podem ser recomendados para substituir o xaxim desfibrado.

Dronk et al. (2012) avaliaram o desenvolvimento de clones de orquídea BLC Nan Chang Silk 'Olimpic Torch', por 24 meses, em oito substratos tendo como base a fibra de xaxim; fibra de coco; casca de pinus decomposta; carvão vegetal e combinações entre eles. Os autores concluíram que todos os substratos avaliados podem ser utilizados eficientemente como alternativos ao xaxim.

Valencia (2009) comparou o uso de: 1) caroços de açaizeiro, após fervura; 2) fibra de coco industrializada; 3) fibras do caule de açaizeiro e; 4) serragem, no cultivo das orquídeas Brassia chloroleuca Barb. Rodr. e Sobralia macrophylla Rchb.. A autora concluiu que, para ambas as espécies, em três, dos quatro parâmetros 
avaliados, os resultados foram estatisticamente iguais.

A revisão bibliográfica mostra que existem substratos que, dependendo do sistema de irrigação e da adição de adubos, podem ser melhorados, com alguns alcançando desempenho superior ao do xaxim desfibrado. Mostra ainda que, o desempenho do substrato está associado à espécie cultivada sobre ele.

Durante o presente estudo notou-se que os substratos com caroços de açaizeiro foram rapidamente consumidos por pequenos animais que aceleraram a sua decomposição. Isto possivelmente facilitou a adsorção de água o que manteve as plantas vivas, avaliadas no primeiro ano, até a avaliação do segundo ano (Tabela 1). A maioria das orquídeas, e entre elas os dendrobiuns, vegetam melhor em substratos com boa aeração que foi diminuída com a decomposição. No entanto, existem espécies que vegetam bem em substratos orgânicos bem decompostos como as dos gêneros Phalaenopsis e Paphiopedilun e para elas, os caroços decompostos poderiam vir a se um componente de substrato de uso potencial.

Orquídeas é um grupo de plantas com distribuição por quase todos os biomas do planeta, portanto possuidoras das mais diversas estratégias de sobrevivência que se possa imaginar. 0 fato dos substratos testados como alternativos ao pó de xaxim não tenha sido superior a ele, a possibilidade de virem a ser usados em outras espécies de orquídeas não deve ser descartada. O trabalho de Valencia (2009) mostrou isto, pois sobre caroços de açaizeiro, mesmo sem adubação, foi possível o desenvolvimento das orquídeas $B$. chloroleuca e S. macrophylla.

Assis et al. (2005) conseguiram que substratos, feitos com base em mesocarpo de coco industrializado, tivessem melhor desempenho que 0 xaxim desfibrado, no cultivo de D. nobile, porém associados à aplicação mensal de 
adubo foliar (NPK 10-10-10 na concentração de $\left.1 \mathrm{gL}^{-1}\right)$ e, a cada noventa dias, à adubação orgânica (utilizando farinha de osso e torta de mamona 1:1 na dosagem de $1 \mathrm{~g}$ /vaso). Yamakami et al. (2006) ao comparar o xaxim desfibrado entre outros substratos, com a fibra de coco, também observaram efeito similar no cultivo de C. labiata x C. forbesii, aplicando o mesmo esquema de adubação. A adubação foliar básica aplicada no presente experimento é de uso corrente entre os orquidófilos, mas ela possivelmente não surtiu efeito como observado por aqueles porque, eles a usam associada à aplicação de adubos orgânicos ricos em nitrogênio com o "bokashi" e a mistura de torta de manona: farinha de ossos. Isto sugere que, o desempenho dos substratos testados no presente trabalho poderia vir a ser melhorados pela adição de fertilizantes orgânicos.

Devido à proibição da exploração do xaxim e a alta disponibilidade de cocosverdes, caroços de açaizeiro e mais recentemente de palmiteiro, sugere-se que melhorias nas formulações e no manejo das plantas cultivadas sobre os substratos que os usem como base, continuem sendo buscadas. Como sugestão para futuros experimentos tem-se: a inclusão de componentes para manter a aeração (no caso dos caroços de açaizeiro); aplicação de adubos (orgânicos e minerais associados); tipo de vaso; e regime de irrigação. Deve ser considerado ainda que, a combinação entre tais fatores deve ser coligada à espécie a ser cultivada.

\section{CONCLUSÃO}

O resultado do presente trabalho corrobora que substratos feitos com caroços de palmiteiro e fibras de pericarpo de coco lavadas podem ser usados como substitutos do substrato formulado somente com o pó de xaxim ou a mistura de pó de xaxim: caroços de palmiteiro para o Dendrobium nobile. 


\section{REFERÊNCIAS}

AMARAL, T. L.; JASMIM, J. M.; ARAUJO, J. S. P.; THIEBAUT, J. T. L.; COELHO, F. C.; FREITAS, C. B. Adubação de orquídeas em substratos com fibra de coco. Ciência e Agrotecnologia, v. 34, n. 1, p.11-19, 2010.

ASSIS, A. M. de; FARIA, R. T. de; UNEMOTO, L. K.; COLOMBO, L. A. Cultivo de Oncidium baueri Lindley (orchidaceae) em substratosa base de coco. Ciência e Agrotecnologia, v. 32, n. 3, p. $981-985,2008$.

ASSIS, A. M. de; FARIA, R. T. de; COLOMBO, L. A.; CARVALHO, J. F. R. P. de Utilização de substratos à base de coco no cultivo de Dendrobium nobile Lindl. (Orchidaceae). Acta Scientiarum. Agronomy, v. 27, n.2, p.255 - 260, 2005.

BAKER, M.; BAKER, C. O. Orchid Species Culture: Dendrobium. Portland: Editora Timber Press, 1996, 852 p.

COLOMBO, L. A.; FARIA, R. T. de; ASSIS, A. M.; FONSECA, C. de B. Aclimatização de um híbrido de Cattleya em substratos de origem vegetal sob dois sistemas de irrigação. Acta Scientiarum - Agronomy, v. 27, n. 1, p. $145-150,2005$.

DEMATTÊ, J. B. I.; DEMATTÊ, M. E. S. P. Estudos hídricos com substratos vegetais para cultivo de orquídeas epífetas. Pesquisa Agropecuária Brasileira, v.31, n. 11, p.803 - 813, 1996.
DRONK, A. G.; SILVA, A. P. V da; CUQUEL, F. L.; FARIA, R. T. de. Desenvolvimento vegetativo de híbrido de orquídea em diferentes substratos. Semina - Ciências Agrárias, v.33, p.2109 - 2114, 2012.

DYTHAM, C. Choosing and Using Statistics: A Biologist's Guide, 3rd edition, West Sussex: Wiley-Blackwell, 2010, 320 p.

FARIA, R. T. de; REGO, L. DO V.; BERNARDI, A., MOLINARI, $H$. Performance of differents genotypes of brazilian orquids cultivation in alternatives substrates. Brazilian Archives of Biology and Technology, v.44, n.4, p.337 - 342, 2001.

MYERS, R. H.; MONTGOMERY, D. C.; GEOFFREY VINING, G.; ROBINSON, T. J. Generalized linear models with applications in engineering and the sciences, 2nd edition. New Jersey: Editora John Wiley \& Sons Inc., 2010, 521 p.

RODRIGUES, R. B.; LICHTENTHÄLER, R.; ZIMMERMANN, $\quad$ B. $\quad F$.; PAPAGIANNOPOULOS, M.; FABRICIUS, H.; MARX, F. Total oxidant scavenging capacity of Euterpe oleracea Mart. (açaí) seeds and identification of their polyphenolic compounds. Journal of Agricultural and Food Chemistry, v.54, n.12, p.4162 - 4167, 2006.

SOCCOL, J. J.; VENTURIERI, G.A, PEDROTTI, E. L. Stems age, nitrogen 
fertilizer and salicylic acid application in cutting induction of noble dendrobium orchid of the Yamamoto series cultivars. Ornamental Horticulture, v.23, n.2, p.131137, 2017.2 DOl: http://dx.doi.org/10.14295/oh.v23i2.884

SORACE, M.; FARIA, R. T. de; FONSECA, I. B.; YAMAMOTO L. Y.; SORACE, M. A. F.; Substratos alternativos ao xaxim no cultivo do híbrido Cattleya intermedia $\mathrm{X}$ Hadrolaelia purpurata (Orchidaceae). Semina - Ciências Agrárias, v.30, p.771 778, 2009.

TAVARES, G. dos S.; HOMMA, A.K.O. Comercialização do açaí no estado do Pará: alguns comentários. Observatorio de la Economía Latinoamericana, Brasil, v. 211 (septiembre), 2015. Disponível em: <http://www.eumed.net/cursecon/ecola t/br/15/acai-para.html>.

TREVISAN, A. C. D.; FANTINI, A. C.; SCHMITT-FILHO, A. L.; FARLEY, J. Market for Amazonian açaí (Euterpe oleraceae) stimulates pulp production from Atlantic Forest juçara berries (Euterpe edulis). Agroecology and Sustainable Food Systems, v. 39, n.7, p.762-781. 2015.

VALENCIA, W. H. Avaliação de substratos orgânicos no cultivo de orquídeas nativas da APA ilha do Combu, Belém, Pará, 2009. 46 f. Tese (Mestrado em Gestão de Recursos Naturais e Desenvolvimento Local na Amazônia) - Universidade Federal do Pará, Belém 2009.
VENTURIERI, G. A.; PICKSCIUS, F. J. Propagation of nobile dendrobium (Dendrobium nobile Lindl.) by cutting. Acta Scientiarum Agronomy, v.35, n.4, p.501-504, 2013.2 DOl: http://dx.doi.org/10.4025/actasciagron.v $35 i 4.15198$

YAMAKAMI, J. K.; FARIA, R. T. de; ASSIS, A. M. de; REGO-OLIVEIRA, L. do V. Cultivo de Cattleya Lindley (Orchidaceae) em substratos alternativos ao xaxim. Acta Scientiarum - Agronomy, v.28, n.4, p.5233 - 526, 2006. 